\title{
Nonlinear Impact of Edge Localized Modes on Carbon Erosion in the Divertor of the JET Tokamak
}

\author{
A. Kreter, ${ }^{1 *}$ H. G. Esser, ${ }^{1}$ S. Brezinsek, ${ }^{1}$ J. P. Coad,${ }^{2}$ A. Kirschner, ${ }^{1}$ W. Fundamenski, ${ }^{2}$ V. Philipps, ${ }^{1}$ \\ R. A. Pitts, ${ }^{3}$ and A. Widdowson ${ }^{2}$ \\ ${ }^{1}$ Institut für Energieforschung - Plasmaphysik, Forschungszentrum Jülich, Association EURATOM-FZJ, \\ Trilateral Euregio Cluster, Germany \\ ${ }^{2}$ EURATOM-UKAEA Fusion Association, Culham Science Centre, Abingdon, United Kingdom \\ ${ }^{3}$ CRPP, Association EURATOM-EPFL, Lausanne, CH-1015, Switzerland
}

(Received 15 January 2008; published 30 January 2009)

\begin{abstract}
The impact of edge localized modes (ELMs) carrying energies of up to $450 \mathrm{~kJ}$ on carbon erosion in the JET inner divertor is assessed by means of time resolved measurements using an in situ quartz microbalance diagnostic. The inner target erosion is strongly nonlinearly dependent on the ELM energy: a single $400 \mathrm{~kJ}$ ELM produces the same carbon erosion as ten $150 \mathrm{~kJ}$ events. The ELM-induced enhanced erosion is attributed to the presence of codeposited carbon-deuterium layers on the inner divertor target, which are thermally decomposed under the impact of ELMs.
\end{abstract}

PACS numbers: 28.52.Fa, 52.35.Py, 52.40.Hf, 52.55.Fa

One of the key issues for the success of the International Thermonuclear Experimental Reactor (ITER) project [1] will be the heat flux handling capability of the plasmafacing components (PFCs). The ITER baseline operating scenario invokes the high confinement $(H)$ mode [2] to achieve burning plasma conditions. One of the characteristic features of the $H$ mode with respect to the low confinement $(L)$ mode is the formation of a transport barrier at the plasma edge. The resulting high pressure gradient leads to quasiperiodic magnetohydrodynamic relaxations of the barrier known collectively as edge localized modes (ELMs) [3]. The largest of these events (type-I ELMs), usually associated with high quality $H$ modes, release substantial fractions $\Delta W_{\mathrm{ELM}}$ of the plasma stored energy $W\left(\Delta W_{\text {ELM }} / W\right.$ of up to $\sim 10 \%$; $W \sim 350$ MJ estimated for ITER) across the magnetic separatrix into the scrape-off layer (SOL). In the SOL, a major fraction of this energy is transported to the divertor, where magnetic field lines terminate on the target plates. The induced target erosion is likely to be severe, leading to unacceptably short divertor target lifetime [4,5]. Even assuming that ELMs can be mitigated such that thresholds for severe damages are not exceeded [6,7], some residual activity is always likely to remain. Investigations of specific carbon and tungsten materials proposed for ITER PFCs have resulted in ELM loads being restricted to $0.5 \mathrm{MJ} / \mathrm{m}^{2}$ [8].

Carbon based materials can withstand extremely high heat loads. This is one reason why carbon fiber composite (CFC) is currently being considered for ITER as material in the area of strongest plasma-surface interaction-the socalled strike point (SP) regions on the divertor targets [1]. One serious disadvantage of carbon for ITER is the redeposition of eroded carbon in the form of amorphous layers containing high quantities of hydrogenic isotopes (the deuterium and tritium constituting the plasma fuel in a fusion reactor). Particular attention must be paid to the codeposited layers growing in areas shadowed from the direct plasma impact. These remote locations may turn out to be inaccessible to cleaning techniques and could result in a reservoir of accumulated tritium. This is a particularly serious concern for ITER, where the quantity of stored tritium will be limited to $1 \mathrm{~kg}$ by nuclear safety restrictions [1].

The experimental program of JET as the worldwide largest operating tokamak is primarily focused on the development of ITER relevant scenarios in terms of confinement physics as well as plasma-wall interaction [9]. Large ELMs in JET can carry an energy of up to $\Delta W_{\text {ELM }} \sim$ $1 \mathrm{MJ}$ over times of several $100 \mu \mathrm{s}$ and an ELM wetted area of the divertor of $\sim 1 \mathrm{~m}^{2}$, leading to peak power fluxes to the divertor targets of up to $\sim 1 \mathrm{GW} / \mathrm{m}^{2}[10,11]$. JET is equipped with an all-carbon first wall and operates mostly with deuterium as the plasma fuel, although it is the only tokamak in existence capable of fueling with tritium. High levels of tritium retention were observed during the deuterium-tritium experiments of 1997 , when $H$-mode discharges with large ELMs were used to produce record values of fusion power [12]. The majority of tritium was found codeposited with carbon in the remote region of the inner divertor leg on a series of water cooled louvres through which gas is exhausted to the cryopumps [13]. The deposition pattern observed at the inner louvre suggested that the carbon transport in the inner divertor is mainly line-of-sight. Both simple particle balance models [13] and sophisticated Monte Carlo simulations of impurity transport $[14,15]$ failed, by at least 1 order of magnitude, to reproduce the high level of codeposition in the inner louvre. Since this modeling was performed for plasma parameters averaged over both inter-ELM and ELM phases, it was speculated that ELMs could increase 


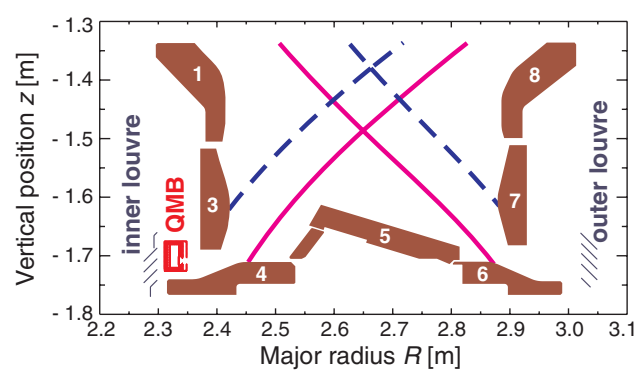

FIG. 1 (color online). Poloidal cross section of the MarkII-HD divertor deployed during the JET campaigns of 2005-2007 with numbers as used in the text to denote the CFC divertor target tiles. The dashed line is the separatrix of the magnetic field configuration common to the pulses in which the QMB data of Fig. 2 are obtained. The full line indicates the configuration chosen for the QMB database used in Figs. 3 and 4. The intersection points of each separatrix with the tiles are the respective strike points.

the amount of carbon eroded from the inner target and transported to the louvre region [13,14]. Until recently, however, no compelling evidence has been found for the additional ELM driven carbon transport. This Letter provides that evidence.

The persistent observation of strongly asymmetric carbon deposition in the JET divertor has triggered the recent development of in situ plasma-wall interaction diagnostics including quartz microbalance (QMB) systems capable of measuring the amount of deposited material from shot to shot [16]. A distinctive feature of the JET QMBs is the protective shutter allowing a time window, typically of a few seconds duration, to be defined in any given discharge. This feature permits the isolation of specific temporal regions, for example, the ELMing $H$-mode phases of interest to this Letter. A QMB measures the net amount of deposited material, namely, the gross deposition on the detector less erosion due to the incident deuterium flux. In this Letter, the amount of deposition on the QMB located at the inner divertor louvre (Fig. 1) is expressed as areal densities of carbon atoms $D_{\mathrm{C}}$. The sensitivity limit of the system is $D_{\mathrm{C}} \approx 1 \cdot 10^{15} \mathrm{C} / \mathrm{cm}^{2}$. For a density of deposited layer of $1 \mathrm{~g} / \mathrm{cm}^{3}$ it corresponds to $\approx 0.2 \mathrm{~nm}$ or about one monolayer of deposited film.

Among the many aspects of carbon transport in the divertor that can be studied with the new QMB system, the effect of ELMs has turned out to be one of the most significant. A striking example of the ELM effect was observed in a pair of discharges with similar plasma conditions but different ELM behavior, as illustrated in Fig. 2 . Both discharges had an input power of $21 \mathrm{MW}$ and employed a magnetic field geometry such that the inner SP was positioned on the vertical tile 3 (Fig. 1). The only discharge actuator modified between the two pulses was the external gas fueling, which is known to strongly influence the ELM energy $\Delta W_{\text {ELM }}$ and frequency $f_{\text {ELM }}$ on JET, with unfuelled discharges producing the largest ELMs [10]. This influence is attributed to the changes in the neoclassical pedestal collisionality $\nu_{\text {ped }}^{*}$ (neo) [4]. A moderate deuterium fueling with a rate of $1.2 \times 10^{22}$ atoms $/ \mathrm{s}$ in the first discharge resulted in a pedestal collisionalitiy of $0.08-0.15$ and zero fueling in the second in $\nu_{\text {ped }}^{*}($ neo $)=$ $0.02-0.06$, close to the projected ITER baseline value of 0.06 . In the first pulse, ELMs with $\Delta W_{\mathrm{ELM}} \approx 300 \mathrm{~kJ}$ at $f_{\mathrm{ELM}} \approx 10 \mathrm{~Hz}$ produced a net-deposition on the QMB in the inner louvre of $D_{\mathrm{C}} \approx 4 \times 10^{15} \mathrm{C} / \mathrm{cm}^{2}$ during its $0.8 \mathrm{~s}$ exposure. Switching off the gas fueling in the second pulse resulted in large but infrequent ELMs $\left(\Delta W_{\mathrm{ELM}} \approx 700 \mathrm{~kJ}\right.$, $f_{\mathrm{ELM}} \approx 3 \mathrm{~Hz}$ ). In this pulse, the QMB exposure time window captured the time period between two large ELMs. The deposition and erosion of the QMB were balanced $\left(D_{\mathrm{C}} \approx 0\right)$ within the accuracy of the system, thus showing that the carbon erosion from the target in the inner divertor decreases dramatically in the absence of large ELMs.

To extract a meaningful ELM scaling from the QMB data covering the period 2005-2007, only pulses meeting

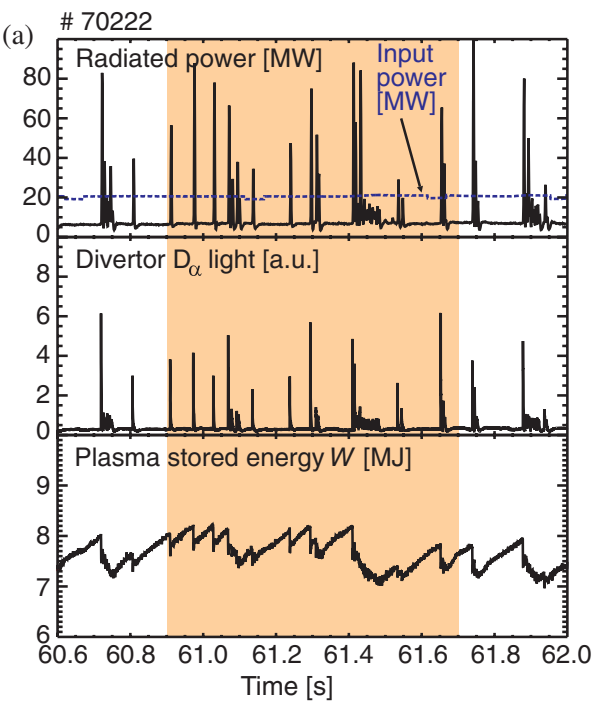

FIG. 2 (color online). A pair of discharges with similar main plasma parameters (see text) but different ELMs. Spikes on signals of the radiated power and the divertor $D_{\alpha}$ light emission are signatures of ELMs, each associated with a sudden drop of the plasma stored energy. The first event in (b) illustrates the definition of the ELM energy drop $\Delta W_{\mathrm{ELM}}$. The time window of the QMB exposure is indicated by shaded region. (a) Discharge with moderate gas fueling. (b) Pulse without fueling. 


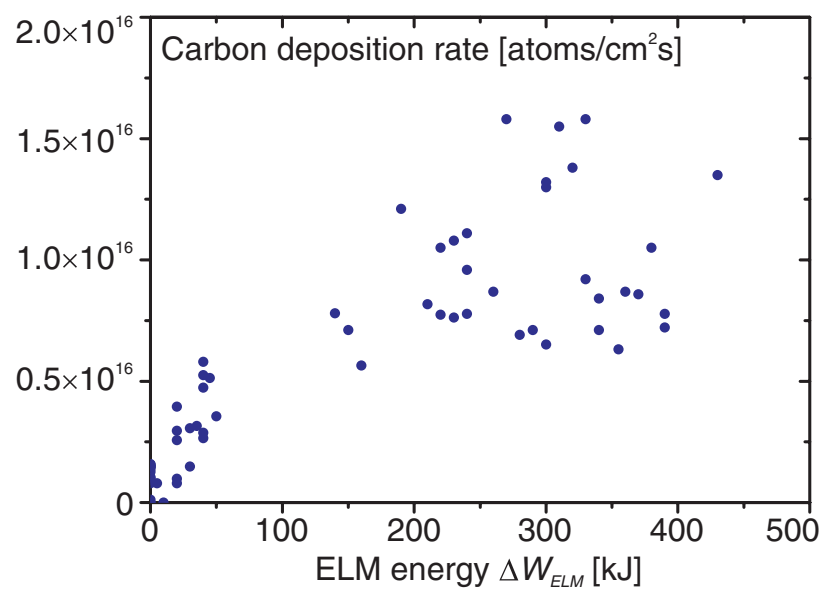

FIG. 3 (color online). Carbon deposition rate of the QMB in the inner louvre as function of ELM stored energy drop.

the following criteria are considered for analysis: (i) exposures occurring only during the phase of stationary plasma conditions; (ii) constant magnetic geometry in the divertor, choosing a configuration with inner SP on horizontal target tile 4; see Fig. 1. This latter condition ensures line-of-sight to the position of the inner divertor louvre, corresponding to magnetic field configurations employed during the deuterium-tritium experiments of 1997. Sixtynine pulses fulfill these selection rules, with 51 in the $H$ mode and 18 in the $L$ mode.

Figure 3 compiles the carbon deposition rates on the inner QMB as a function of $\Delta W_{\mathrm{ELM}}$ for the selected pulses. Although quasiperiodic, ELMs are typically comprised of a range of energies and frequencies in an otherwise stable discharge [4]. As illustrated in Fig. 2, there will often be a mixture of different ELM sizes during a single QMB exposure. The abscissa value in Fig. 3 is therefore defined as the mean energy of all events which occurred during the respective QMB exposure with energies $>80 \%$ of the largest $\Delta W_{\mathrm{ELM}}$. The mixture of different ELMs, along with different plasma parameters and properties of the target surface in respective discharges, can also explain the scatter of the data points, which is significantly larger than the accuracy of the deposition measurements. Nevertheless, the data have a clear trend for $\Delta W_{\mathrm{ELM}}<$ $100 \mathrm{~kJ}$ with deposition rates increasing significantly with ELM energy. For larger ELMs the dependence of deposition rates on ELM energy becomes less pronounced. The frequency of large type-I ELMs decreases with increasing ELM energy [10]. A smaller number of large ELMs appears to largely compensate the increase of erosion induced by these ELMs.

A further step in the analysis can be obtained by estimating the deposition on the QMB due to single ELMs and deriving a fit function providing an analytic scaling for the deposition per ELM (Fig. 4). The abscissa in Fig. 4 has the same definition as in Fig. 3, namely, the mean energy of the largest events occurring during the given QMB exposure. For all data points in Fig. 3, the deposition rates were

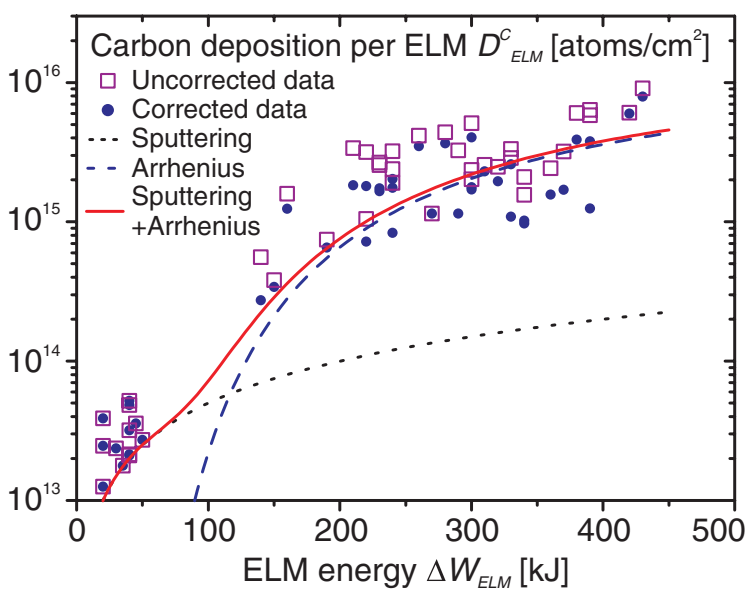

FIG. 4 (color online). Amount of carbon deposited on the inner louvre QMB per single ELM as function of ELM stored energy drop. ( $\square$ ) Original uncorrected data; $(\bigcirc)$ data corrected for the influence of smaller ELMs; (solid line) self-consistent fit function comprising (dotted line) linear term for physical sputtering and (dashed line) Arrhenius term for thermal decomposition.

divided by the number of these events to obtain the deposition per ELM $D_{\mathrm{ELM}}^{\mathrm{C}}$ (open squares in Fig. 4). Each of the derived data points, however, includes a contribution due to additional, smaller ELMs within the exposure period. These contributions are quantified and subtracted from the original data points by fitting the following equation to the data set:

$$
D_{\mathrm{ELM}}^{\mathrm{C}}=D_{S}^{\mathrm{C}} \Delta W_{\mathrm{ELM}}+D_{a}^{\mathrm{C}} \exp \left(-W_{a} / \Delta W_{\mathrm{ELM}}\right),
$$

where $D_{a}^{\mathrm{C}}$ and $W_{a}$ are fit coefficients and $D_{S}^{\mathrm{C}}$ is fixed to $5 \times$ $10^{11} \mathrm{C} / \mathrm{cm}^{2} \mathrm{~kJ}$ according to the argument given below. Using an iterative fitting procedure a self-consistent function with fit coefficients $D_{a}^{C}=1.97 \times 10^{16} \mathrm{C} / \mathrm{cm}^{2}$ and $W_{a}=680 \mathrm{~kJ}$ is obtained. The correction of the original data with this fit function delivers the data set, which, in turn, yields the same fit function. Both the original and the corrected data are shown along with the fit function in Fig. 4.

The fit formula is the sum of two terms containing $\Delta W_{\text {ELM }}$. The first, $D_{S}^{\mathrm{C}} \Delta W_{\mathrm{ELM}}$, represents physical sputtering of carbon by deuterium ions during ELMs (assuming a linear relationship between the impinging ion flux and the ELM energy). Coefficient $D_{S}^{\mathrm{C}}$ is estimated for plasma parameters in the divertor target vicinity typical for an ELM of a few $100 \mathrm{~kJ}$ [11], a sputtering yield of $1.5 \%$ [17] and the QMB geometry with respect to the inner SP position. QMB deposition induced by small ELMs with $\Delta W_{\mathrm{ELM}}<50 \mathrm{~kJ}$ is reasonably well reproduced by physical sputtering. However, for larger ELMs deposition reveals a clear nonlinear behavior with significantly higher values, which cannot be assigned to physical sputtering. Chemical erosion of carbon can be neglected on the basis that at the elevated target surface temperatures $\left(>1000^{\circ} \mathrm{C}\right)$ and high 
fluxes $\left(>10^{20} \mathrm{~cm}^{-2} \mathrm{~s}^{-1}\right)$, characteristic for the large ELMs, the erosion yield is $<0.1 \%$ [18].

The nonlinear behavior of $\mathrm{QMB}$ deposition for $\Delta W_{\mathrm{ELM}}>\sim 100 \mathrm{~kJ}$ is well described by the second term in the fit formula, the ubiquitous Arrhenius function. The fact that the process obeys an Arrhenius-like scaling implies a thermal origin and suggests that the strong increase in observed QMB carbon deposition rate beyond a given $\Delta W_{\mathrm{ELM}}$ is due to thermal decomposition of carbon in the ELM impact regions. In this case, $W_{a}$ is identified as the activation energy of the process. Infrared thermography of the JET divertor target capable of resolving ELM-induced heat fluxes [19] has demonstrated that, even for ELMs of up to $\approx 700 \mathrm{~kJ}$, the surface temperature rarely exceeds $1500^{\circ} \mathrm{C}$ [20] and never reaches temperatures above $2200^{\circ} \mathrm{C}$, where thermal sublimation of graphite becomes the dominant erosion mechanism [17]. However, the ELM energies characteristic of JET appear to be sufficient to trigger thermal decomposition of codeposited carbondeuterium layers in the inner divertor. The high sensitivity of the layers is reflected in spectroscopic observations of carbon emission lines in the JET divertor [21]. The thermal decomposition becomes the dominant mechanism of erosion at $\Delta W_{\mathrm{ELM}} \sim 100 \mathrm{~kJ}$, corresponding to $\sim 100 \mathrm{~kJ} / \mathrm{m}^{2}$ in terms of energy density, which is well below the revised limit for ELMs in ITER of $500 \mathrm{~kJ} / \mathrm{m}^{2}$ [8]. The ELMinduced enhanced erosion is therefore likely to occur in ITER.

The mechanism for carbon layer thermal decomposition in the JET divertor is still a matter of discussion. Spikes in surface temperature induced by large ELMs appear to be relevant for radiation-enhanced sublimation (RES), a thermal effect distinctive for carbon materials, reviewed in [17]. However, the erosion yield of RES decays with increasing particle flux $[17,22]$ and, extrapolated to the ELM conditions, would be below the value for physical sputtering.

A possible mechanism of enhanced erosion of the codeposited layers is thermal release of a large family of hydrocarbons, as it was observed during heat treatment of hydrogenated carbon layers [23]. However, the ionization length of thermally released carbon atoms and hydrocarbon molecules for plasma conditions during large ELMs is $<1 \mathrm{~mm}$, so that the majority of particles will be promptly redeposited and will not find their way to the QMB location. Another possible scenario is the ELM driven formation of carbon particle clusters from the target surface layers. These dust particles can easily overcome the distance between the inner SP and QMB of $\sim 10 \mathrm{~cm}$ [24], in agreement with the observed line-of-sight character of the carbon transport to the inner louvre.

In conclusion, the data derived from the QMB diagnostic are the first direct evidence of ELM-induced enhanced carbon erosion in the JET inner divertor. Even when divertor target conditions are far from the threshold for carbon sublimation, ELMs enhance erosion of codeposited carbon-deuterium layers in a nonlinear manner, provoking much faster material dislocation towards shadowed areas. The observed Arrhenius behavior of this erosion points to a thermally assisted process, strongly suggesting that large ELMs induce thermal decomposition of deposited layers on the inner divertor target surfaces. The result explains the large amount of carbon and hydrogenic isotopes typically observed in the remote areas of the inner divertor of JET, in particular, after the 1997 deuterium-tritium campaign.

This work, supported by the European Communities, was carried out within the framework of the European Fusion Development Agreement. This work was done under the JET-EFDA work programme [9].

*a.kreter@fz-juelich.de

[1] A. Loarte et al., Nucl. Fusion, 47 S203 (2007).

[2] F. Wagner et al., Phys. Rev. Lett. 49, 1408 (1982).

[3] H. Zohm, Plasma Phys. Controlled Fusion 38, 105 (1996).

[4] A. Loarte et al., Plasma Phys. Controlled Fusion 45, 1549 (2003).

[5] G. Federici et al., Plasma Phys. Controlled Fusion 45, 1523 (2003).

[6] T. E. Evans et al., Phys. Rev. Lett. 92, 235003 (2004).

[7] Y. Liang et al., Phys. Rev. Lett. 98, 265004 (2007).

[8] A. Zhitlukhin et al., J. Nucl. Mater. 363-365, 301 (2007).

[9] M. L. Watkins et al., Proceedings of the 21st International Conference on Fusion Energy, Chengdu, 2006 [International Atomic Energy Agency (IAEA), Vienna, 2006]. (All members of the JET-EFDA Collaboration appear in the appendix of this paper.)

[10] A. Loarte et al., Phys. Plasmas 11, 2668 (2004).

[11] R. A. Pitts et al., Nucl. Fusion 47, 1437 (2007).

[12] M. Keilhacker, M.L. Watkins, and JET Team, J. Nucl. Mater. 266-269, 1 (1999).

[13] J.P. Coad, P. L. Andrew, and A. T. Peacock, Phys. Scr. T81, 7 (1999).

[14] J. N. Brooks et al., J. Nucl. Mater. 313-316, 424 (2003).

[15] A. Kirschner et al., Plasma Phys. Controlled Fusion 45, 309 (2003).

[16] H. G. Esser et al., J. Nucl. Mater. 337-339, 84 (2005).

[17] W. Eckstein and V. Philipps, in Physical Processes of the Interaction of Fusion Plasmas with Solids, edited by W. Hofer and J. Roth (Academic Press, San Diego, 1996), p. 93.

[18] J. Roth et al., Nucl. Fusion 44, L21 (2004).

[19] T. Eich et al., Plasma Phys. Controlled Fusion 49, 573 (2007).

[20] R. A. Pitts, Bull. Am. Phys. Soc. 52, http://meetings. aps.org/Meeting/DPP07/Event/69758 (2007).

[21] S. Brezinsek et al., J. Nucl. Mater. 337-339, 1058 (2005).

[22] Y. Ueda et al., J. Nucl. Mater. 227, 251 (1996).

[23] K. Maruyama, W. Jacob, and J. Roth, J. Nucl. Mater. 264, 56 (1999).

[24] S. I. Krasheninnikov et al., Phys. Plasmas 11, 3141 (2004). 